\title{
Modulation of Working Memory and Resting-State fMRI by tDCS of the Right Frontoparietal Network
}

\author{
Monika Pupíková $\mathbb{D}^{1,2}$ Patrik Šimko $\mathbb{D}^{1,2}$ Martin Gajdoš $\mathbb{D}^{1,3}$ and Irena Rektorová ${ }^{1}{ }^{1,4}$ \\ ${ }^{1}$ Central European Institute of Technology-CEITEC, Masaryk University, Applied Neuroscience Research Group, \\ Brno, Czech Republic \\ ${ }^{2}$ Faculty of Medicine, Masaryk University, Brno, Czech Republic \\ ${ }^{3}$ Central European Institute of Technology-CEITEC, Masaryk University, Multimodal and Functional Neuroimaging \\ Research Group, Brno, Czech Republic \\ ${ }^{4}$ First Department of Neurology, Faculty of Medicine, Masaryk University and St. Anne's University Hospital, Brno, Czech Republic
}

Correspondence should be addressed to Irena Rektorová; irena.rektorova@fnusa.cz

Received 11 February 2021; Revised 25 June 2021; Accepted 9 July 2021; Published 26 July 2021

Academic Editor: Carlo Cavaliere

Copyright (@) 2021 Monika Pupíková et al. This is an open access article distributed under the Creative Commons Attribution License, which permits unrestricted use, distribution, and reproduction in any medium, provided the original work is properly cited.

\begin{abstract}
Many cognitive functions, including working memory, are processed within large-scale brain networks. We targeted the right frontoparietal network (FPN) with one session of transcranial direct current stimulation (tDCS) in an attempt to modulate the cognitive speed of a visual working memory task (WMT) in 27 young healthy subjects using a double-blind crossover design. We further explored the neural underpinnings of induced changes by performing resting-state fMRI prior to and immediately after each stimulation session with the main focus on the interaction between a task-positive FPN and a task-negative default mode network (DMN). Twenty minutes of $2 \mathrm{~mA}$ anodal tDCS was superior to sham stimulation in terms of cognitive speed manipulation of a subtask with processing of objects and tools in unconventional views (i.e., the higher cognitive load subtask of the offline WMT). This result was linked to the magnitude of resting-state functional connectivity decreases between the stimulated FPN seed and DMN seeds. We provide the first evidence for the action reappraisal mechanism of object and tool processing. Modulation of cognitive speed of the task by tDCS was reflected by FPN-DMN cross-talk changes.
\end{abstract}

\section{Introduction}

The integrity and connectivity of large-scale brain networks were shown to play a crucial role in the individual performances of distinct cognitive tasks-namely, in the interplay between the task-positive frontoparietal network (FPN) and the "task-negative" default mode network (DMN) [1-3]. In accordance with this notion, we showed in young healthy subjects (HY) that manipulation of the excitability of the posterior parietal cortex (that is part of the FPN) with repetitive transcranial magnetic stimulation (rTMS) led to remote cognitive task-induced BOLD signal decreases of the DMN along with enhanced cognitive speed [4]. While the frontoparietal cortices contribute to adaptive behavior control via the flexible encoding of task demands and desired outcomes and the top-down modulation of processing in other brain regions [5], the DMN is involved in mind wandering and various aspects of self-referential internally directed processing, and its activity is suppressed by most goal-directed tasks $[2,6]$. Disruption to both the FPN and the DMN is a common finding in Alzheimer's disease and Parkinson's disease, i.e., the most common neurodegenerative brain diseases leading to cognitive decline in aging populations [7-10]. Some authors have suggested that the magnitude and direction of task-related hemodynamic responses within regions engaged in the task-positive and task-negative networks are related to the intrinsic connectivity patterns between these networks [11], and it may therefore be plausible to use resting-state functional connectivity (rs-FC) measures between the major task-positive and task-negative brain networks to examine the neural correlates of cognitive modulation induced by targeted noninvasive brain stimulation. 
Among various noninvasive stimulation techniques, transcranial direct current stimulation (tDCS) was proposed as an inexpensive and easily administered method for experimental and potentially also for clinical use in an attempt to modulate brain function [12]. The tDCS causes only very mild side effects such as itching and tingling [13], and unlike rTMS, it seems optimal even for supervised home care [14]. tDCS applies a weak direct electric current through two electrodes placed over the scalp with the goal of modulating underlying cortical excitability [15]. The effects of tDCS lead to subthreshold changes in membrane potentials towards depolarization/hyperpolarization [16]. The tDCS efficacy for modulating cognitive accuracy and speed is still in question [17-19]. Moreover, little is known about the neural underpinnings of tDCS-induced cognitive changes in the healthy brain.

In the current study, we used tDCS with the aim of modulating visual working memory (WM) performance. WM refers to the limited capacity system for online temporary storage and simultaneous manipulation of information to be employed in ongoing processing $[20,21]$. It is related to higher-order cognitive skills such as multitasking or learning [22] therefore central to the execution of a variety of daily functions. One influential way how to conceptualize working memory is a multicomponent system comprising modalityspecific temporary memory systems, which store information and refresh memory traces, and a supervisory system (so-called central executive) that is tasked with various executive functions such as focusing and switching attention, coordinating the storage systems as well as activating the contents within long-term memory [23]. The loss of WM efficiency related to either aging or a pathological process is hypothesized to have various neurobiological roots, among which altered functioning of the FPN plays an important role [24-26]. We used a right-sided frontoparietal montage based on the results of our previous visual WMT fMRI research (matching objects from conventional vs. unconventional views) performed in patient groups with mild cognitive impairment due to a neurodegenerative brain disease [27]. The patients displayed cognitive deficits along with deficient involvement of the right-sided multimodal (domain general) frontoparietal regions, suggesting altered top-down processing; the activation of cortical areas within the ventral and dorsal visual pathways (i.e., bottom-up processing) remained preserved as compared to controls [27]. We hypothesized that tDCS of the right frontoparietal regions would alter the efficiency and cognitive speed of the same visual WMT by modulating the top-down control of visual processing, even in healthy young individuals. We further hypothesized that the tDCS-induced cognitive speed modulations of the offline WMT would be reflected by intervention-induced changes in the intrinsic functional connectivity between the FPN and the DMN.

\section{Materials and Methods}

2.1. Subjects. Participants were recruited from a university environment (we targeted mainly students or early career researchers) in the age range between 18 and 35 years. Partic- ipants did not receive any monetary compensation. Exclusion criteria were the presence of any psychiatric or neurological disorder, cognitive deficit based on the Montreal Cognitive Assessment (cut-off score 26 points), and drug or alcohol abuse. All subjects had normal or corrected-tonormal visual acuity. Written informed consent was obtained from all subjects prior to the experiment. The study was performed according to the guidelines of the Declaration of Helsinki and approved by the local ethics committee. The study was registered at clinicaltrials.gov with reference ID NCT04134195.

2.2. Study Protocol. In this double-blind controlled trial with a crossover design, the subjects were tested in two different experimental sessions, each corresponding to the active or sham stimulation conditions (see Figure 1). Prior to the experimental sessions, each subject underwent T1 MRI sequence scanning to enable individual targeting of tDCS montage (for further details, see below) and practiced the experimental tasks in order to reduce the practice effects, which are most prominent between the first and the second sessions [28]. Each session lasted approximately 2 hours and included a visual object matching task (VOMT) [27] and a resting-state fMRI examination-both performed before and immediately after each tDCS session (active, sham). The tDCS was run outside the scanner simultaneously with another visual working memory task (online WMT) in order to enhance the stimulation aftereffects [29, 30], see Figure 1. The order of experimental conditions was counterbalanced across subjects.

2.3. Visual Working Memory Tasks. Throughout the study, we used two different visual working memory tasks. A visual-object matching task (VOMT) [27] was our main behavioral outcome task, and it was performed before and immediately after tDCS (i.e., the offline VOMT).

The task consists of multiple successive paired images of common objects. The second image of each pair is either the same or different from the first image (different object identity or object orientation). Participants are instructed to respond as quickly as possible by pressing a YES button if the second object of the paired images is the same as the first object (regardless of spatial orientation) or by pressing a $\mathrm{NO}$ button if the second object is different. Each trial comprised the following sequence: a mask stimulus ( $1 \mathrm{~s})$, followed by the picture of the first object ( $1 \mathrm{~s})$, followed by a mask (1 s), followed by the picture of the second object ( $1 \mathrm{~s})$, followed by a mask ( $1 \mathrm{~s})$, ending with a fixation cross $(5 \mathrm{~s})$. Conditions are presented in a randomized order (see Figure 1(b)). Overall, participants viewed 40 trials in total, 20 trials in each condition. The task takes 7 minutes to accomplish. We collected the number of correct responses and reaction times (RT) of both conditions-conventional view (lower difficulty level) condition and unconventional view (higher difficulty level) condition with rotated object views. We used different versions of the task for every session, balanced in difficulty. Based on previous tDCS study results in young participants $[31,32]$, we specifically focused on the unconventional view of object condition subtask of the VOMT (i.e., the subtask 

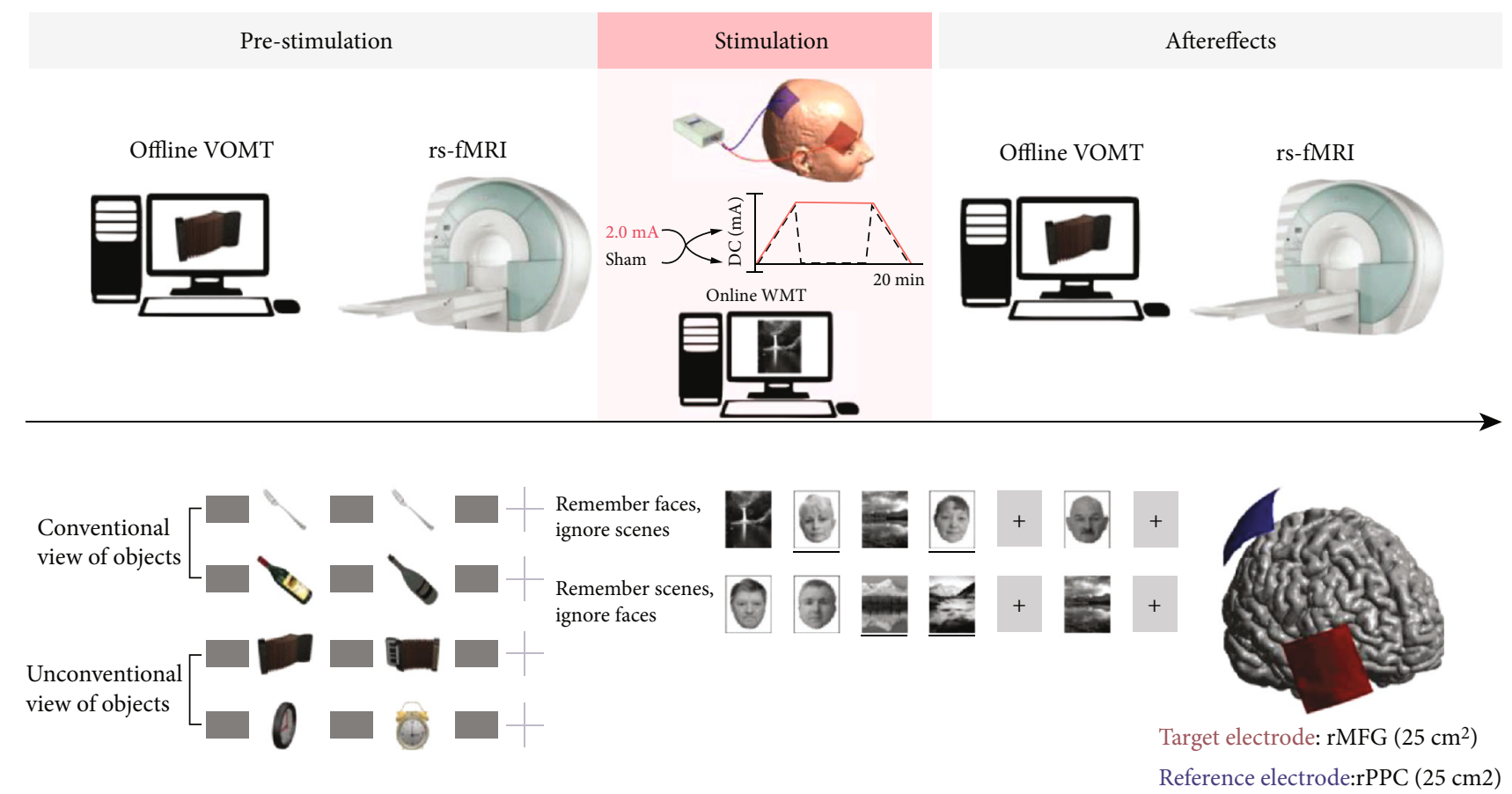

Figure 1: Experimental design and methods. (a) The crossover design involved two sessions with real $2 \mathrm{~mA}$ stimulation/sham tDCS with concurrent working memory task. Prior to and after the stimulation, participants performed a visual object matching task and underwent resting-state fMRI. (b) Offline VOMT-subjects respond whether the two consecutive objects are the same or different by pressing the YES/NO button in two difficulty levels (conventional view of objects-lower difficulty level; unconventional view of objects-higher difficulty level). (c) Online WMT-subjects view a block of faces and scenes $(2+2$, randomized order) preceded by a specific command on how to react to a probe which follows each block. Subjects respond whether the probe is consistent/inconsistent with the prior instruction by pressing the YES/NO button. (d) Montage for real and sham tDCS.

with a higher cognitive load) and on processing speed rather than task accuracy as our primary outcome measure.

The online WMT involved a working memory task with faces and outdoor scenes adopted from Gazzaley et al. [33] which was performed during the stimulation session (active, sham). The task consisted of two subtasks in which aspects of visual information were maintained constant while the target instruction changed. Participants were instructed to attend to one particular category and ignore irrelevant distractor category, so there were two kinds of instructions: (1) "remember faces and ignore scenes" vs. (2) "remember scenes and ignore faces." During each trial, participants viewed a series of four images - two faces and two scenes - in a randomized order. Images were presented sequentially for $800 \mathrm{msec}$. After a retention period of 9 seconds, a probe was presented (consistent with the prior instruction) and participants responded whether or not the probe was present in the foregoing series. The task was performed for the whole duration of the stimulation, thus 20 minutes. In total, 48 trials were presented, and conditions 1 and 2 were randomized into 4 blocks consisting of 10 trials balanced in condition (see Figure $1(\mathrm{c})$ ). The number of correct responses and reaction times (RT) was collected.

2.4. tDCS Stimulation Parameters. tDCS was performed through a battery-driven stimulator (DC-Stimulator Plus, neuroConn $\mathrm{GmbH}$, Germany). The anode was positioned over the right middle frontal gyrus ( $\mathrm{rMFG}$; $\mathrm{MNI}=4440$
-10) with the cathode over the right posterior parietal cortex (rPPC, MNI = $30-55$ 52). We used the T1 MRI scan-based frameless stereotactic neuronavigation targeting with Brainsight 2 , to specify the exact location of the electrode center in each individual. With this electrode montage, we aimed to modulate the excitability of the right frontoparietal multimodal regions based on a cortical parcellation atlas described in Yeo et al. [34]. The current of $2 \mathrm{~mA}$ was delivered using two rubber electrodes $(5 \times 5 \mathrm{~cm})$ for 20 minutes, with initial ramp-up and final ramp-down phases. The electrode was held in place by conductive paste (Ten20 Conductive Paste gel, Weaver and Company). The sham stimulation was applied with the same settings, but the stimulator was turned off after 30 seconds. The impedance was controlled by the device throughout the session limited by the voltage at $26 \mathrm{~V}$, not exceeding $15 \mathrm{k} \Omega$. An excess of limits would have led to an automatic termination of stimulation.

2.5. Magnetic Resonance Imaging. MRI data were acquired with a 3.0 T Magnetom Siemens Prisma. We acquired T1 MPRAGE sequence (TR $1570 \mathrm{~ms}$; TE $2.45 \mathrm{~ms}$; voxel size 1 $\times 1 \times 1 \mathrm{~mm}$; FoV $256 \times 226 \mathrm{~mm}$; flip angle $8^{\circ}$; 160 transversal slices) in all participants to navigate the stimulation (as described above). A subgroup of 22 participants underwent resting-state fMRI (rs-fMRI; $n=22$; TR $850 \mathrm{~ms}$; TE $35.2 \mathrm{~ms}$; voxel size $2 \times 2 \times 2 \mathrm{~mm}$; FoV $208 \mathrm{~mm}$; flip angle $45^{\circ}$; 80 transversal slices; 700 scans; multiband factor 8 ) prior to and immediately after each tDCS condition. 


\subsection{Data Analysis}

2.6.1. Analysis of Behavioral Data. We recorded the number of correct responses and reaction times (RT) in both tasks (see in Results). Based on the results of previous studies in young participants, including our own research $[4,35,36]$, we predicted ceiling effects for task accuracy; we therefore focused on RT changes. Normality of data was assessed with Kolmogorov-Smirnov tests. Paired $t$-tests were used to compare differences (RT post-RT baseline) in VOMT performance between stimulation conditions (active vs. sham). The baseline RT in VOMT was measured before each sham or active stimulation session and calculated as their mean. The slopes of the reaction time learning curves ( $\beta$ RT) for the online WMT were compared between active and sham groups using paired $t$-tests. We further correlated online and offline effects by comparing $\Delta \mathrm{RT}$ of VOMT and $\beta \mathrm{RT}$ of the online WMT. Data were corrected for learning effects during sessions and analyzed with SPSS 24.0 software.

2.6.2. Analysis of Resting-State fMRI Data. The mean rs-FC was assessed between the anode seed ( $\mathrm{rMFG}$ ) and the major DMN seeds (i.e., spheres with $r=6 \mathrm{~mm}$ ) using six coordinates as described previously [2], see Figure 2. Representative mean seed signals were extracted, and correlation matrix was calculated for each subject. Pearson's correlation coefficients were converted using Fisher's $r$-to- $z$ transformation to $z$ values. The average connectivity between the anode seed and DMN seeds was calculated as the mean of $z$ values for each seed pair. Because of the nonnormality of the data, Wilcoxon signed-rank tests were used to assess the tDCSinduced changes in the rs-FC. We further correlated differences of RT outcomes (RT post-RT baseline) with differences of $z$ values of the connectivity changes (FC post-FC baseline) separately for real/sham condition.

\section{Results}

3.1. Demographic Data. The sample consisted of 31 righthanded young healthy volunteers. Altogether, data from four subjects were excluded: two participants did not finish the stimulation protocol due to health problems unrelated to the study protocol, and the data from two subjects were not fully recorded due to technical problems. The final dataset consists of 27 subjects, mean age $27 \pm 4.1$ years, 11 men and 16 women. A subsample of 22 subjects also underwent fMRI acquisition (mean age: $24.7 \pm 4.6 ; 10$ men and 12 women).

3.2. Behavioral Results. All subjects tolerated the stimulation well and mentioned only minor side effects (tingling, itching under the electrode) that faded away during the stimulation. As predicted, we observed a ceiling effect and low variability in correct responses for both the baseline VOMT subtask (median, IQR: 95\%; 90-95\%) and the baseline online WMT task (95\%; 87-98\%). Regarding RT changes, we found a significant difference between the real and sham stimulation conditions only for the VOMT subtask with more cognitively demanding unconventional views of objects, i.e., our subtask of interest $\left(\Delta \mathrm{RT}_{\text {real }}=-0.015 \mathrm{~s} ; \Delta \mathrm{RT}_{\text {sham }}=0.026 \mathrm{~s} ; p=0.049\right)$,

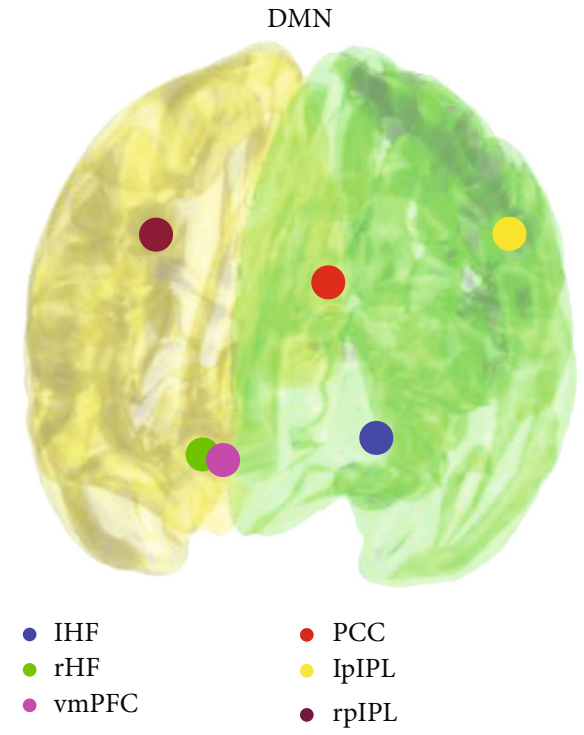

FIgURe 2: Seeds used for the rs-fMRI data analysis: default mode network. $1 / \mathrm{r}$ a IPL $=$ left/right anterior inferior parietal lobule; $1 / \mathrm{rHF}=$ left/right hippocampal formation; $\mathrm{vmPFC}=$ ventromedial prefrontal cortex; $\mathrm{PCC}=$ posterior cingulate cortex; $\mathrm{l} / \mathrm{rIPL}=$ left/right posterior inferior parietal lobule.

see Figure 3(a). As predicted, results for the whole VOMT (both subtasks with lower and higher cognitive load) were not significant $\left(\Delta \mathrm{RT}_{\text {real }}=-0.007 \mathrm{~s} ; \Delta \mathrm{RT}_{\text {sham }}=0.02 \mathrm{~s} ; p=\right.$ 0.085). There was no significant difference in $\beta$ RT for the online WMT task between active and sham conditions (see Supplementary Figure 1). Despite this negative effect, we observed that $\beta \mathrm{RT}$ for the online WMT correlated with $\Delta$ RT for the VOMT subtask with a higher cognitive load in the real but not in the sham tDCS condition $\left(r_{\text {real }}=0.501\right.$, $\left.p=0.018 ; r_{\text {sham }}=0.071, p=0.754\right)$, see Figures 3(b) and 3(c).

3.3. fMRI Results. We found no differences between stimulation conditions (real and sham tDCS) in changes of the rMFG-DMN connectivity ( $\Delta$ rMFG-DMN) (mean $z$ values: $\Delta \mathrm{rMFG}-\mathrm{DMN}_{\text {real }}=0.006, \Delta \mathrm{rMFG}-\mathrm{DMN}_{\text {sham }}=0.028, p=$ 0.149 ); however, we observed a significant positive correlation between the $\Delta \mathrm{RT}$ in the VOMT subtask with unconventional object view and $\Delta$ rMFG-DMN for the real tDCS $(r=0.459$ and $p=0.032)$, see Figure 4 . No such correlation was observed for the sham stimulation condition $(r=0.275$; $p=0.216)$.

\section{Discussion}

In this study, we demonstrated that in young universitybased healthy subjects, a single session of real tDCS over the right frontoparietal regions of the FPN was superior to sham tDCS in terms of affecting the cognitive speed of the cognitively more demanding subtask of the WMT (with unconventional views of objects). The impact of real tDCS on task accuracy could not be studied due to the ceiling performance of the task already at the baseline. Our results are consistent with previous studies in which only the cognitive speed of the WMT was enhanced after prefrontal tDCS 


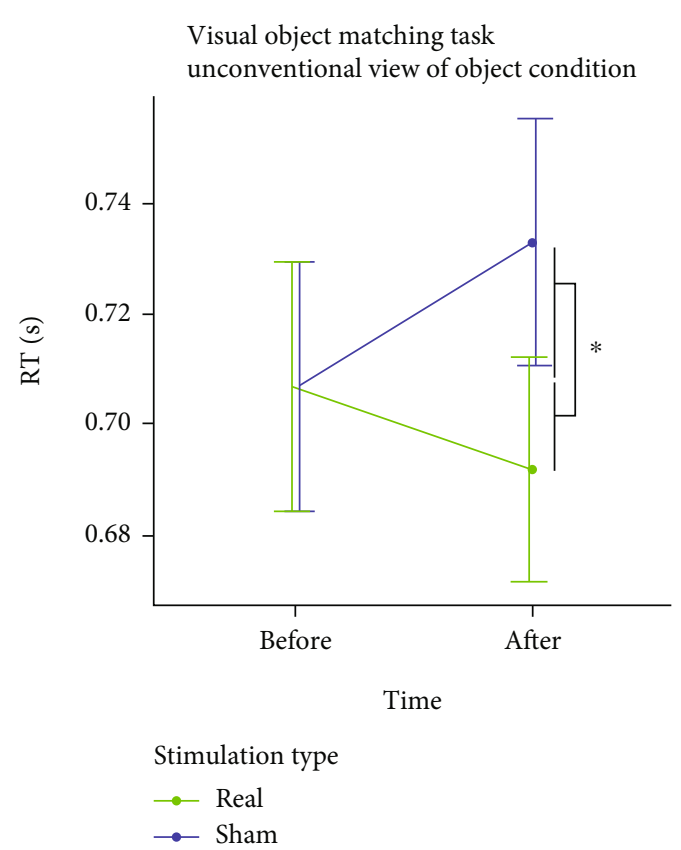

(a)

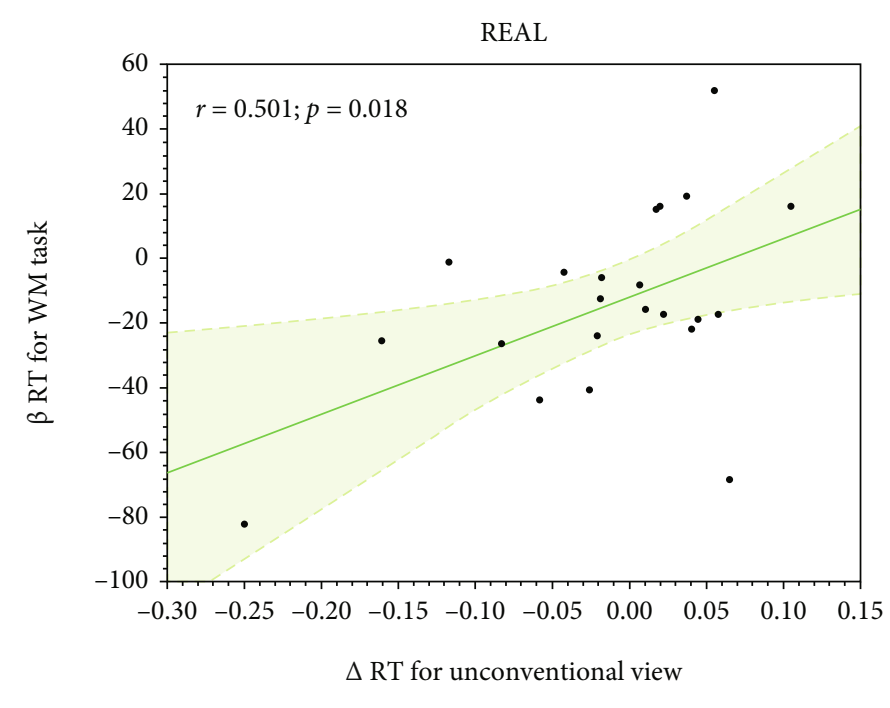

(b)

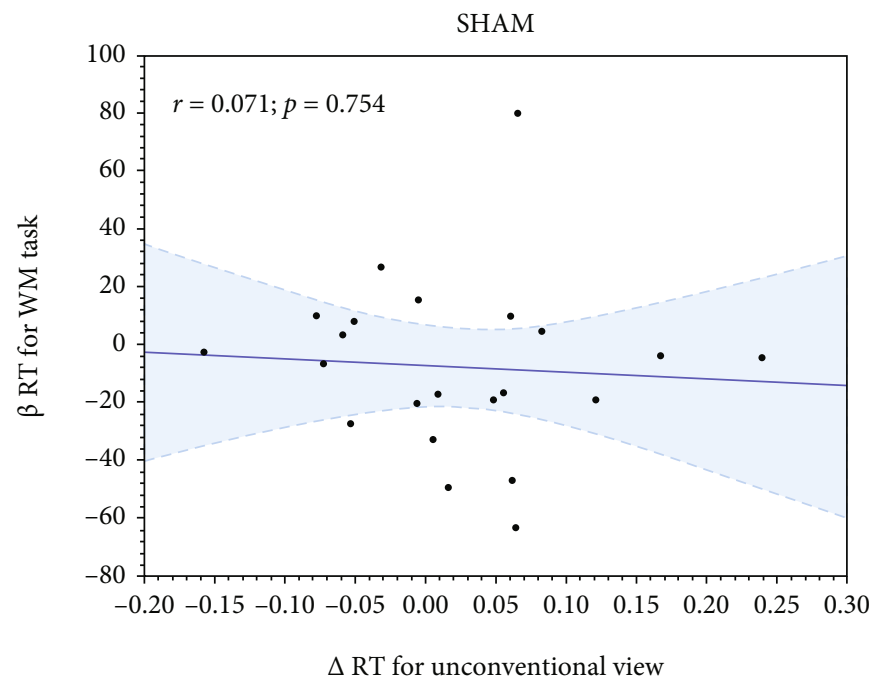

(c)

Figure 3: Behavioral results (a) offline VOMT (mean \pm SE); (b) correlation of behavioral results online and offline tasks after tDCS (b) real and (c) sham ${ }^{*} p<0.05$.

stimulation in young healthy volunteers [37-41]. Two metaanalyses also indicated a favorable enhancement of reaction times with small but significant effect sizes in healthy populations as compared to accuracy improvement in patient samples with baseline WM deficits $[42,43]$. However, null results of tDCS on cognition have also been reported [35, 44]. Among the factors that seem to influence the efficacy of tDCS in HY, the increased task load was shown to play an important role [31, 32, 37, 45].

Of note, the most common target of previous tDCS studies was the dorsolateral prefrontal cortex (DLPFC) $[18,42$, 46]. Our anode was positioned in the anterior part of the right middle frontal gyrus, and the cathode was placed in the right posterior parietal cortex. This right-sided frontoparietal rather than bifrontal montage was based on our fMRI study with the same visual WMT [27]. In that study, deficient engagement of the right-sided FPN regions involved in working memory tasks and in the top-down control of visual processing [47-50] led to impaired task performance in patient groups as compared to healthy controls. In the current study, manipulating the excitability of the same right-sided FP regions by $\mathrm{tDCS}$ affected the processing speed of the WMT subtask with a higher cognitive load even in young healthy subjects. Taken together, using task fMRI for precise tDCS electrode placement may strengthen stimulation-induced cognitive aftereffects on that task. Future studies should 


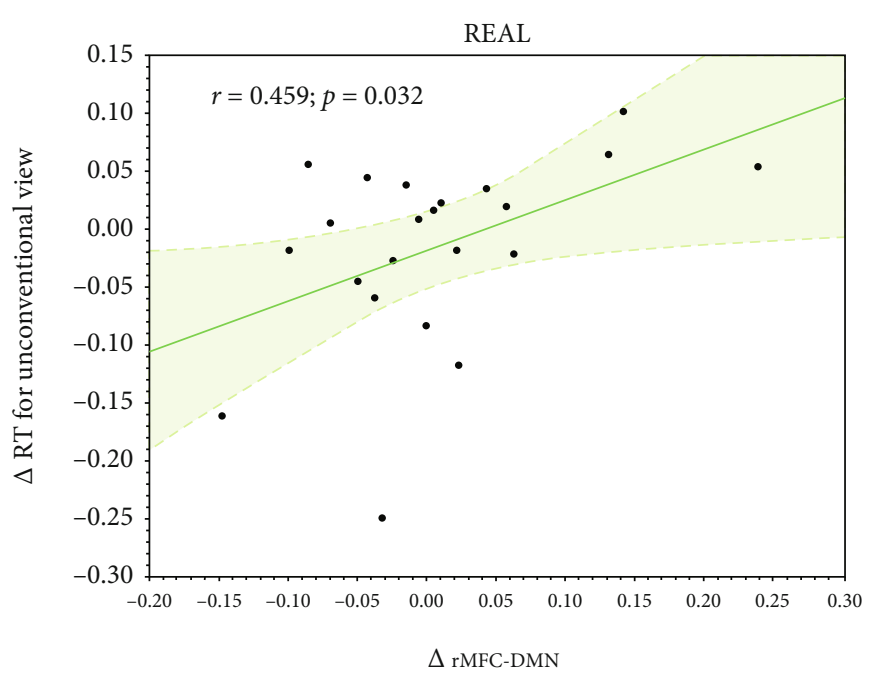

(a)

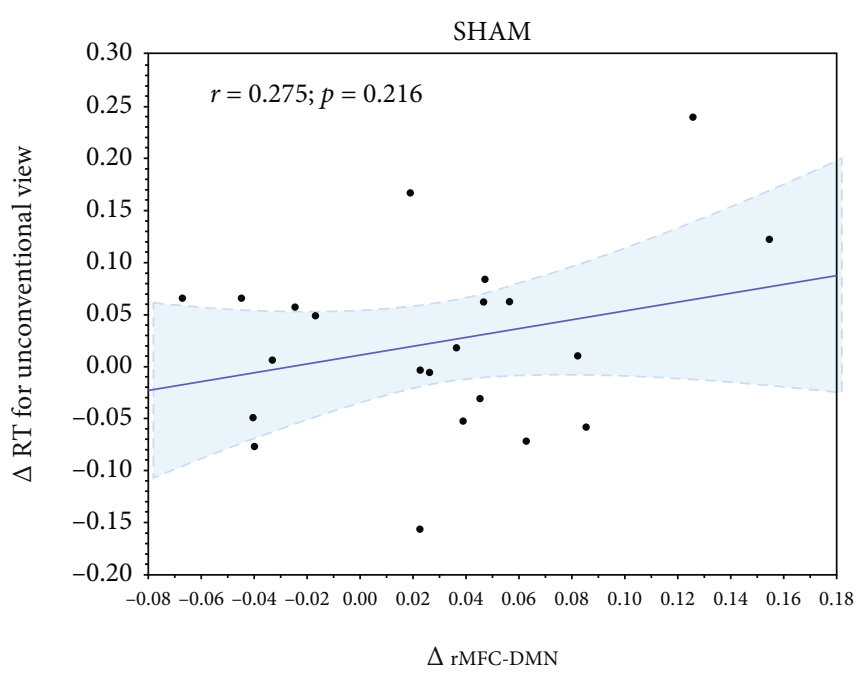

(b)

FIGURE 4: Correlation of behavioral results with rMFG-DMN rs-connectivity for (a) real and (b) sham conditions.

explore whether such a specific modulation of the given WMT could also transfer to modulation of other cognitive tasks or even cognitive domains. We believe this might be possible particularly when domain-general regions of the FPN, known to be involved in top-down control of cognitive processing, are stimulated as in the current study.

We did not observe any significant modulation of the online WMT by tDCS. This finding is in line with the results of a meta-analysis by Hill et al. [43] that reported that anodal tDCS enhanced RTs of the offline WMTs in a healthy population; no significant effects were found for the online tasks. Despite insignificant results for the online WMT, we observed that the RT decreases of the online WMT in the real tDCS condition were associated with more pronounced cognitive speed changes of the offline VOMT subtask of interest. This finding may have clinical implications; future studies should examine whether the online cognitive speed modulation could predict offline aftereffects of multiple sessions of tDCS and thus help choose optimal candidates for longterm tDCS.

Our results may also be viewed in a neurocognitive theorem of object-tool processing and recognition. The right FPN (the main target of our tDCS stimulation) is actively implicated in high-level executive functions [2]. Recent evidence indicates its specific role in object and tool recognition [47, $48,50-52]$ through the concept of "action reappraisal." Action reappraisal is a multidimensional cognitive process combining multiple sources of information (e.g., semantic knowledge, mechanical knowledge, and sensorimotor knowledge) processed in a recursive semantic-to-mechanical-tomotor "cascade" [53], subserved by a dynamic interaction of complex brain networks (particularly the interplay between the frontoparietal and occipitotemporal networks), thus providing a generalizable and in everyday context usable object representations [51, 52, 54-56]. A series of behavioral studies employing eye tracking provided evidence that higher-level information is activated earlier than lower-level perceptual information and can affect a visuoattentional pattern in which the objects are processed, although the magnitude of top-down processes is modulated by context and expectations [54-56]. The action reappraisal concept, as part of the reasoning-based framework [53], provides an alternative to the well-established embodied-cognition approach which suggests that object knowledge is constituted by information inscribed within the motor and sensory systems thus stressing the automatic lower-level processing of information [57]. In the current study, we modified the speed of object and tool recognition in the offline VOMT task by targeting the FPN (and the right MFG in particular) by tDCS. We thus provide the first empirical "causal" evidence for the action reappraisal mechanism of object and tool processing. Interestingly, this effect seemed to be modality specific as no significant effects were found for the online WMT comprising faces and scenes stimuli. Notably, other explanations for the modality-specific aftereffects are possible as different effects of offline and online stimulation on various WMTs have been observed [58].

The tDCS-induced rs-FC changes between the anode seed (engaged in the task-positive FPN) and the DMN seeds were not significantly different between the real and sham stimulation conditions. However, our behavioral results correlated with the real tDCS-induced rs-FC changes between the frontal hub of the FPN (our anode seed) and the DMN such that the VOMT subtask speeding was linked to between-network connectivity decreases. Previous studies demonstrated that many cognitive functions, including working memory, are processed within large-scale brain networks and are dependent on their dynamic cross-talk. For example, decreases of functional connectivity between the task-positive FPN and DMN were linked to better performance of a WMT that was further strengthened by online frontal tDCS [59]. Conversely, an increase of rs-FC between the FPN and the DMN was associated with negative cognitive outcomes in healthy subjects and in patient groups $[1,60$, 
61]. Such an increase of the between-network rs-FC could be related to a higher propensity to mind wandering and lapses of attention [62], which may in turn lead to cognitive slowing [63-65]. Here, we show for the first time that manipulating right-sided FPN by tDCS during concurrent cognitive training may modulate the speed of top-down processing of an offline visual WMT (i.e., measured after the intervention and compared to baseline performance) that is linked to the offline rs-FC decreases between task-positive and tasknegative brain networks. Similar brain-behavior associations were recently shown after concurrent WMT training with frontal tDCS, such that decreases of activity in the anterior DMN node correlated with faster responses as measured by offline task-fMRI [66]. However, the results of rs-FC and task-related activations cannot be directly compared.

\section{Conclusion}

We demonstrated cognitive speed modulation of the offline WMT in young healthy subjects that was induced by a single session tDCS coupled with the online cognitive training as compared to online cognitive training alone (coupled with sham tDCS). We provide the first empirical evidence for the action reappraisal mechanism of object and tool processing. The behavioral tDCS-induced changes were correlated with the magnitude of the FPN-DMN cross-talk changes after the real stimulation condition. Therefore, the betweennetwork rs-FC measures may be used to monitor tDCSinduced cognitive aftereffects.

\section{Data Availability}

The datasets used for supporting the findings in this study are available from the corresponding author on reasonable request.

\section{Conflicts of Interest}

The authors declare that there is no conflict of interest regarding the publication of this paper.

\section{Acknowledgments}

We acknowledge the core facility MAFIL of CEITEC supported by the Czech-BioImaging Large RI Project (LM2018129 funded by MEYS CR) for their support with obtaining scientific data presented in this paper. We thank Anne Johnson for English language editing. This project has received funding from a grant from the Czech Ministry of Health NV18-04-00256 (The effect of transcranial direct current stimulation on visual attention in mild cognitive impairment - a combined fMRI and non-invasive brain stimulation study). This publication was written with the support of the Specific University Research (MUNI/A/1465/2020) provided by MŠMT.

\section{Supplementary Materials}

Behavioral results of the online WMT task might be seen in Supplementary Figure 1. (Supplementary Materials)

\section{References}

[1] C. A. M. Kelly, L. Q. Uddin, B. B. Biswal, F. X. Castellanos, and M. P. Milham, "Competition between functional brain networks mediates behavioral variability," NeuroImage, vol. 39, no. 1, pp. 527-537, 2008.

[2] W. Gao and W. Lin, "Frontal parietal control network regulates the anti-correlated default and dorsal attention networks," Human Brain Mapping, vol. 33, no. 1, pp. 192-202, 2012.

[3] M. D. Fox, A. Z. Snyder, J. L. Vincent, M. Corbetta, D. C. Van Essen, and M. E. Raichle, "The human brain is intrinsically organized into dynamic, anticorrelated functional networks," Proceedings of the National Academy of Sciences of the United States of America, vol. 102, no. 27, pp. 9673-9678, 2005.

[4] L. Nováková, M. Gajdoš, and I. Rektorová, “Theta-burst transcranial magnetic stimulation induced cognitive task-related decrease in activity of default mode network: an exploratory study," Brain Stimulation, vol. 13, no. 3, pp. 597-599, 2020.

[5] M. W. Cole, D. S. Bassett, J. D. Power, T. S. Braver, and S. E. Petersen, "Intrinsic and task-evoked network architectures of the human brain," Neuron, vol. 83, no. 1, pp. 238-251, 2014.

[6] M. E. Raichle, "The brain's default mode network," Annual Review of Neuroscience, vol. 38, no. 1, pp. 433-447, 2015.

[7] L. Krajcovicova, R. Marecek, M. Mikl, and I. Rektorova, "Disruption of resting functional connectivity in Alzheimer's patients and at-risk subjects," Current Neurology and Neuroscience Reports, vol. 14, no. 10, p. 491, 2014.

[8] Q. Zhao, H. Lu, H. Metmer, W. X. Y. Li, and J. Lu, "Evaluating functional connectivity of executive control network and frontoparietal network in Alzheimer's disease," Brain Research, vol. 1678, pp. 262-272, 2018.

[9] S. Lehericy, D. E. Vaillancourt, K. Seppi et al., "The role of high-field magnetic resonance imaging in parkinsonian disorders: pushing the boundaries forward," Movement Disorders, vol. 32, no. 4, pp. 510-525, 2017.

[10] L. Anderkova, M. Barton, and I. Rektorova, "Striato-cortical connections in Parkinson's and Alzheimer's diseases: relation to cognition," Movement Disorders, vol. 32, no. 6, pp. 917922, 2017.

[11] M. D. Greicius, B. Krasnow, A. L. Reiss, and V. Menon, "Functional connectivity in the resting brain: a network analysis of the default mode hypothesis," Proceedings of the National Academy of Sciences of the United States of America, vol. 100, no. 1, pp. 253-258, 2003.

[12] W. Y. Hsu, Y. Ku, T. P. Zanto, and A. Gazzaley, "Effects of noninvasive brain stimulation on cognitive function in healthy aging and Alzheimer's disease: a systematic review and metaanalysis," Neurobiology of Aging, vol. 36, no. 8, pp. 23482359, 2015.

[13] A. R. Brunoni, M. A. Nitsche, N. Bolognini et al., "Clinical research with transcranial direct current stimulation (tDCS): challenges and future directions," Brain Stimulation, vol. 5, no. 3, pp. 175-195, 2012.

[14] L. E. Charvet, M. T. Shaw, M. Bikson, A. J. Woods, and H. Knotkova, "Supervised transcranial direct current stimulation (tDCS) at home: a guide for clinical research and practice," Brain Stimulation, vol. 13, no. 3, pp. 686-693, 2020.

[15] M. A. Nitsche and W. Paulus, "Excitability changes induced in the human motor cortex by weak transcranial direct current stimulation," The Journal of Physiology, vol. 527, no. 3, Part 3, pp. 633-639, 2000. 
[16] T. Radman, R. L. Ramos, J. C. Brumberg, and M. Bikson, "Role of cortical cell type and morphology in subthreshold and suprathreshold uniform electric field stimulation _in vitro_," Brain Stimulation, vol. 2, no. 4, pp. 215-228.e3, 2009.

[17] A. Holczer, V. L. Németh, T. Vékony, L. Vécsei, P. Klivényi, and A. Must, "Non-invasive brain stimulation in Alzheimer's disease and mild cognitive impairment-a state-of-the-art review on methodological characteristics and stimulation parameters," Frontiers in Human Neuroscience, vol. 14, 2020.

[18] M. Pupíková and I. Rektorová, "Non-pharmacological management of cognitive impairment in Parkinson's disease," Journal of Neural Transmission, vol. 127, no. 5, pp. 799-820, 2020.

[19] L. Anderková and I. Rektorová, "Noninvasive brain stimulation and implications for nonmotor symptoms in Parkinson's disease," International Review of Neurobiology, vol. 134, pp. 1091-1110, 2017.

[20] A. Baddeley, "Working memory," Current Biology, vol. 20, no. 4, pp. 136-140, 2010.

[21] A. D. Baddeley, G. J. Hitch, and R. J. Allen, "A multicomponent model of working memory," in Working Memory: The State of the Science, pp. 10-43, Oxford university press, 2020.

[22] Z. Shipstead, T. S. Redick, and R. W. Engle, "Does working memory training generalize?," Psychologica Belgica, vol. 50, no. 3-4, pp. 245-276, 2010.

[23] A. Baddeley, "Working memory: theories, models, and controversies," Annual Review of Psychology, vol. 63, no. 1, pp. 1-29, 2012.

[24] V. Zachariou, C. E. Bauer, E. R. Seago, F. D. Raslau, D. K. Powell, and B. T. Gold, "Cortical iron disrupts functional connectivity networks supporting working memory performance in older adults," NeuroImage, vol. 223, article 117309, 2020.

[25] Z. A. Yaple, W. D. Stevens, and M. Arsalidou, "Meta-analyses of the n-back working memory task: fMRI evidence of agerelated changes in prefrontal cortex involvement across the adult lifespan," NeuroImage, vol. 196, pp. 16-31, 2019.

[26] J. P. Trujillo, N. J. H. M. Gerrits, D. J. Veltman, H. W. Berendse, Y. D. van der Werf, and O. A. van den Heuvel, "Reduced neural connectivity but increased task-related activity during working memory in de novo Parkinson patients," Human Brain Mapping, vol. 36, no. 4, pp. 1554-1566, 2015.

[27] N. Němcová Elfmarková, M. Gajdoš, I. Rektorová, R. Mareček, and S. Z. Rapcsak, "Neural evidence for defective top-down control of visual processing in Parkinson's and Alzheimer's disease," Neuropsychologia, vol. 106, pp. 236-244, 2017.

[28] A. Collie, P. Maruff, D. G. Darby, and M. McStephen, "The effects of practice on the cognitive test performance of neurologically normal individuals assessed at brief test-retest intervals," Journal of the International Neuropsychological Society, vol. 9, no. 3, pp. 419-428, 2003.

[29] G. Kronberg, A. Rahman, M. Sharma, M. Bikson, and L. C. Parra, "Direct current stimulation boosts Hebbian plasticity in vitro," Brain Stimulation, vol. 13, no. 2, pp. 287-301, 2020.

[30] N. Nozari, K. Woodard, and S. L. Thompson-Schill, "Consequences of cathodal stimulation for behavior: when does it help and when does it hurt performance?," PLoS One, vol. 9, no. 1, article e84338, 2014.

[31] T. Y. Hsu, C. H. Juan, and P. Tseng, "Individual differences and state-dependent responses in transcranial direct current stimulation," Frontiers in Human Neuroscience, vol. 10, no. $643,2016$.
[32] A. C. Ehlis, F. B. Haeussinger, A. Gastel, A. J. Fallgatter, and C. Plewnia, "Task-dependent and polarity-specific effects of prefrontal transcranial direct current stimulation on cortical activation during word fluency," NeuroImage, vol. 140, pp. 134-140, 2016.

[33] A. Gazzaley, J. W. Cooney, J. Rissman, and M. D'Esposito, "Top-down suppression deficit underlies working memory impairment in normal aging," Nature Neuroscience, vol. 8, no. 10, pp. 1298-1300, 2005.

[34] R. L. Buckner, F. M. Krienen, A. Castellanos, J. C. Diaz, and B. T. T. Yeo, "The organization of the human cerebellum estimated by intrinsic functional connectivity," Journal of Neurophysiology, vol. 106, no. 5, pp. 2322-2345, 2011.

[35] L. E. Mancuso, I. P. Ilieva, R. H. Hamilton, and M. J. Farah, "Does transcranial direct current stimulation improve healthy working memory?: a meta-analytic review," Journal of Cognitive Neuroscience, vol. 28, no. 8, pp. 1063-1089, 2016.

[36] L. Anderkova, D. Pizem, P. Klobusiakova, M. Gajdos, E. Koritakova, and I. Rektorova, "Theta burst stimulation enhances connectivity of the dorsal attention network in young healthy subjects: an exploratory study," Neural Plasticity, vol. 2018, Article ID 3106918, 6 pages, 2018.

[37] Y. J. Wu, P. Tseng, C. F. Chang et al., "Modulating the interference effect on spatial working memory by applying transcranial direct current stimulation over the right dorsolateral prefrontal cortex," Brain and Cognition, vol. 91, pp. 87-94, 2014.

[38] K. E. Hoy, M. R. L. Emonson, S. L. Arnold, R. H. Thomson, J. Daskalakis, and P. B. Fitzgerald, "Testing the limits: investigating the effect of tDCS dose on working memory enhancement in healthy controls," Neuropsychologia, vol. 51, no. 9, pp. 1777-1784, 2013.

[39] F. Teo, K. E. Hoy, Z. J. Daskalakis, and P. B. Fitzgerald, “Investigating the role of current strength in tDCS modulation of working memory performance in healthy controls," Frontiers in Psychiatry, vol. 2, 2011.

[40] D. Keeser, T. Meindl, J. Bor et al., "Prefrontal transcranial direct current stimulation changes connectivity of restingstate networks during fMRI," The Journal of Neuroscience, vol. 31, no. 43, pp. 15284-15293, 2011.

[41] P. G. Mulquiney, K. E. Hoy, Z. J. Daskalakis, and P. B. Fitzgerald, "Improving working memory: exploring the effect of transcranial random noise stimulation and transcranial direct current stimulation on the dorsolateral prefrontal cortex," Clinical Neurophysiology, vol. 122, no. 12, pp. 2384-2389, 2011.

[42] J. Dedoncker, A. R. Brunoni, C. Baeken, and M. A. Vanderhasselt, "A systematic review and meta-analysis of the effects of transcranial direct current stimulation (tDCS) over the dorsolateral prefrontal cortex in healthy and neuropsychiatric samples: influence of stimulation parameters," Brain Stimulation, vol. 9, no. 4, pp. 501-517, 2016.

[43] A. T. Hill, P. B. Fitzgerald, and K. E. Hoy, "Effects of anodal transcranial direct current stimulation on working memory: a systematic review and meta-analysis of findings from healthy and neuropsychiatric populations," Brain Stimulation, vol. 9, no. 2, pp. 197-208, 2016.

[44] J. C. Horvath, J. D. Forte, and O. Carter, "Quantitative review finds no evidence of cognitive effects in healthy populations from single-session transcranial direct current stimulation (tDCS)," Brain Stimulation, vol. 8, no. 3, pp. 535-550, 2015.

[45] I. Papazova, W. Strube, A. Wienert et al., "Effects of $1 \mathrm{~mA}$ and $2 \mathrm{~mA}$ transcranial direct current stimulation on working 
memory performance in healthy participants," Consciousness and Cognition, vol. 83, p. 102959, 2020.

[46] J. Dedoncker, A. R. Brunoni, C. Baeken, and M. A. Vanderhasselt, "The effect of the interval-between-sessions on prefrontal transcranial direct current stimulation (tDCS) on cognitive outcomes: a systematic review and meta-analysis," Journal of Neural Transmission, vol. 123, no. 10, pp. 1159-1172, 2016.

[47] G. Ganis, H. E. Schendan, and S. M. Kosslyn, "Neuroimaging evidence for object model verification theory: role of prefrontal control in visual object categorization," NeuroImage, vol. 34, no. 1, pp. 384-398, 2007.

[48] H. E. Schendan and C. E. Stern, "Where vision meets memory: prefrontal-posterior networks for visual object constancy during categorization and recognition," Cerebral Cortex, vol. 18, no. 7, pp. 1695-1711, 2008.

[49] T. A. Niendam, A. R. Laird, K. L. Ray, Y. M. Dean, D. C. Glahn, and C. S. Carter, "Meta-analytic evidence for a superordinate cognitive control network subserving diverse executive functions," Cognitive, Affective and Behavioral Neuroscience, vol. 12, no. 2, pp. 241-268, 2012.

[50] H. E. Schendan and C. E. Stern, "Mental rotation and object categorization share a common network of prefrontal and dorsal and ventral regions of posterior cortex," NeuroImage, vol. 35, no. 3, pp. 1264-1277, 2007.

[51] R. Ishibashi, G. Pobric, S. Saito, and M. A. L. Ralph, "The neural network for tool-related cognition : an activation likelihood estimation meta-analysis of 70 neuroimaging contrasts," Cognitive Neuropsychology, vol. 33, no. 3-4, pp. 241-256, 2016.

[52] E. Reynaud, M. Lesourd, J. Navarro, and F. Osiurak, “On the neurocognitive origins of human tool use : a critical review of neuroimaging data," Neuroscience and Biobehavioral Reviews, vol. 64, pp. 421-437, 2016.

[53] F. Osiurak, G. Federico, M. A. Brandimonte, E. Reynaud, and M. Lesourd, "On the temporal dynamics of tool use," Frontiers in Human Neuroscience, vol. 14, 2020.

[54] G. Federico and M. A. Brandimonte, "Tool and object affordances: An ecological eye-tracking study," Brain and Cognition, vol. 135, article 103582, 2019.

[55] G. Federico and M. A. Brandimonte, "Looking to recognise : the pre-eminence of semantic over sensorimotor processing in human tool use," Scientific Reports, vol. 10, 2020.

[56] G. Federico, F. Osiurak, M. A. Brandimonte, and G. Federico, "Hazardous tools : the emergence of reasoning in human tool use," Psychological Research, vol. 43, 2021.

[57] L. J. Buxbaum and S. Kalénine, "Action knowledge, visuomotor activation, and embodiment in the two action systems," Annals of the New York Academy of Sciences, vol. 1191, no. 1, pp. 201-218, 2010.

[58] M. Zivanovic, D. Paunovic, U. Konstantinovic, K. Vulic, J. Bjekic, and S. R. Filipovic, "The effects of offline and online prefrontal vs parietal transcranial direct current stimulation (tDCS) on verbal and spatial working memory," Neurobiology of Learning and Memory, vol. 179, article 107398, 2021.

[59] L. M. Li, I. R. Violante, R. Leech et al., "Brain state and polarity dependent modulation of brain networks by transcranial direct current stimulation," Human Brain Mapping, vol. 40, no. 3, pp. 904-915, 2019.

[60] H. Baggio, B. Segura, R. Sala-Llonch et al., "Cognitive impairment and resting-state network connectivity in Parkinson's disease," Human Brain Mapping, vol. 36, no. 1, pp. 199-212, 2015.
[61] M. Hampson, N. Driesen, J. K. Roth, J. C. Gore, and R. T. Constable, "Functional connectivity between task-positive and task-negative brain areas and its relation to working memory performance," Magnetic Resonance Imaging, vol. 28, no. 8, pp. 1051-1057, 2010.

[62] C. A. Godwin, M. A. Hunter, M. A. Bezdek et al., "Functional connectivity within and between intrinsic brain networks correlates with trait mind wandering," Neuropsychologia, vol. 103, pp. 140-153, 2017.

[63] V. Axelrod, X. Zhu, and J. Qiu, "Transcranial stimulation of the frontal lobes increases propensity of mind- wandering without changing meta-awareness," Scientific Reports, vol. 8, no. 1, p. 15975, 2018.

[64] J. C. Mcvay and M. J. Kane, "Does mind wandering reflect executive function or executive failure? Comment on Smallwood and Schooler (2006) and Watkins (2008)," Psychological Bulletin, vol. 136, no. 2, pp. 188-197, 2010.

[65] D. R. Thomson, P. Seli, D. Besner, and D. Smilek, "On the link between mind wandering and task performance over time," Consciousness and Cognition, vol. 27, pp. 14-26, 2014.

[66] K. Abellaneda-Pérez, L. Vaqué-Alcázar, R. Perellón-Alfonso et al., "Differential tDCS and tACS effects on working memory-related neural activity and resting-state connectivity," Frontiers in Neuroscience, vol. 13, 2020. 